\title{
49 SCOLIOSIS: MUSCLE IMBALANCE AND TREATMENT
}

Zoran Šarčević Novi Sad Health Care Centre, Sports Medicine Centre, Novi Sad, Serbia

10.1136/bjsm.2010.078725.49

The aim of this study was to detect muscle imbalance on both sides of the backbone, and with muscle strength training, to correct this imbalance and, as a final result, to eliminate scoliosis of the spine. Forty-seven children ( 32 girls and 15 boys), aged 10-16 years, were examined. Muscle strength tests showed that $\mathrm{m}$. quadratus lumborum and $\mathrm{m}$. gluteus medius on the convex side of the spinal curvature appeared to be weaker compared to concave side, and $\mathrm{m}$. erector spine lumbalis and $\mathrm{m}$. multifidus on the concave side of the spinal curvature appeared to be weaker compared to convex side. In the thoracic region, $m$. trapezius medius, $\mathrm{m}$. trapezius distalis and $\mathrm{m}$. serratus anterior on the convex side appeared to be weaker compared to concave side, and $\mathrm{m}$. rhomboideus on the concave side appeared to be weaker compared to convex side. Apart from intense muscle training

for strengthening of weak back muscle groups, all patients were instructed to do the abdominal muscle exercises.

This treatment lasted from 2 to 6 months. Using this method of intense muscle training, scoliosis was eliminated with a non-surgical treatment. 